\title{
Diagnóstico da área de Requisitos de Software usando IDEAL, CMMI e SCAMPI: Um Estudo de Caso
}

\author{
George Marsicano Corrêa, Rejane M. da Costa Figueiredo, Káthia M. de Oliveira
}

Pró-Reitoria de Pós-Graduação e Pesquisa - Mestrado em Gestão do Conhecimento e da Tecnologia da Informação (MGCTI) - Universidade Católica de Brasília (UCB), Caixa Postal: 70790-160, Brasília - DF - Brasil

georgemarsicano@brturbo.com, \{rejane,kathia\} @ucb.br

\begin{abstract}
A company from Brasilia began software process improvement with the requirement area, in order to grow the quality of his products and reduce the re-work. To do this, it was used the diagnostic stage of IDEAL approach to identify the present situation on the requirement area considering the specific actions defined by CMMI model for the process areas - Requirements Management and Requirements Development - and the assessment criteria defined by SCAMPI ${ }^{S M}$ Method. As result, the weakness, its causes and consequences were highlighted. In this way it was possible to set good practices in the Requirement and Development Requirement areas.
\end{abstract}

Resumo. Com o intuito de aumentar a qualidade de seus produtos e diminuir o re-trabalho, uma empresa de Brasília iniciou a melhoria do seu processo de software tendo como foco a área de requisitos. Para isso, foi utilizada a fase de Diagnóstico da abordagem IDEAL para mapear a situação atual da área de requisitos, nas práticas específicas propostas pelo modelo CMMI para as áreas de processo - Gerenciamento de Requisitos e Desenvolvimento de Requisitos - e na definição dos critérios de avaliação do Método $S C A M P I^{S M}$. Como resultado, foram identificados pontos fracos, suas causas e impactos no processo da empresa que inviabilizavam a prática bem estabelecida da Gerência e Desenvolvimento de Requisitos.

\section{Introdução}

Segundo o Instituto de Engenharia de Software (SEI - Software Engineering Institute) a qualidade de um sistema ou produto é altamente influenciada pela qualidade do processo utilizado para desenvolvê-lo e mantê-lo [SEI, 2001]. Porém, para que o processo possa efetivamente exercer tal influência é essencial que o mesmo seja melhorado continuamente. Para uma iniciativa de melhoria de processo é necessário definir: uma abordagem de melhoria, um modelo de referência e um método para orientar a criação de uma estratégia de avaliação baseada no modelo de referência.

Nesse sentido, diversas iniciativas estão sendo tomadas com o intuito de melhorar a qualidade dos processos de software nas empresas. Em Santos [2004] é relatada uma experiência com um programa de melhoria focado na Gestão da Qualidade. Pires [2004] apresenta o planejamento e a implantação do SW-CMM nível 2, tendo como base o ciclo de melhoria PDCA. Carvalho [2001] define uma estratégia para a implantação de uma gerência de requisitos para a melhoria de processos de 


\section{Simpósio Brasileiro de Qualidade de Software}

software, baseadas nas boas práticas existentes na empresa, na cultura da organização e nas características de seus clientes. Sommervile [2005] expõe um estudo empírico realizado na indústria com o intuito, entre outros, de avaliar os benefícios que a melhoria no processo de engenharia de requisitos poderia trazer para a organização.

Nesse contexto, a empresa Brasília (nome fictício adotado neste artigo), iniciou à melhoria do seu processo de desenvolvimento de software, tendo como foco a área de requisitos, por julgar esta uma área estratégica para a empresa. Acredita-se que a melhoria do processo de requisitos irá influenciar diretamente no aumento da qualidade de seus produtos e na diminuição do re-trabalho.

Para alcançar esses objetivos foi utilizado a abordagem IDEAL (Initiating, Diagnosing, Establishing, Acting, Learning) como guia para implantar a melhoria de processo desejada. Associado a $\boldsymbol{I D E A L}$ foi utilizado o modelo de referência, a versão 1.1 do CMMI (Capability Maturity Model Integration), para a melhoria dos processos da organização. O foco foi em duas Áreas de Processo, da categoria de engenharia: Gerenciamento de Requisitos e Desenvolvimento de Requisitos. Como método de avaliação para a definição dos critérios de avaliação e caracterização das práticas

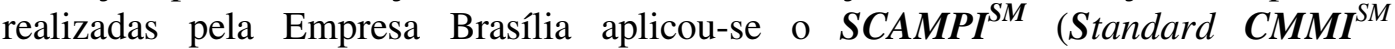
Appraisal Method for Process Improvement).

Neste artigo é apresentado como foi realizada a avaliação considerando a fase de Diagnóstico do $\boldsymbol{I D E A L}$. Nas próximas seções deste trabalho são apresentadas breves revisões da abordagem de melhoria de processos IDEAL (Seção 2), do modelo de referência CMMI e das Áreas de Processo de Gerenciamento de Requisitos e Desenvolvimento de Requisitos (Seção 3), do método de avaliação $\boldsymbol{S C A M P I}^{\boldsymbol{S M}}$ (Seção 4). Na Seção 5, apresenta-se a Implantação da fase de Diagnóstico da Abordagem IDEAL. Finalizando, as conclusões e propostas de trabalhos futuros (Seção 6).

\section{Abordagem de Melhoria de Processos - IDEAL}

A IDEAL é uma abordagem de melhoria de processo, elaborada pelo SEI em colaboração com a Hewlett-Packard Company [McFEELEY, 1996] a partir das idéias mais importantes dos movimentos de qualidade industrial das últimas décadas [DEMING, 1990].

A abordagem propõe cinco fases para a realização de um ciclo de melhoria de processos [McFEELEY, 1996]: (i) iniciação, na qual são definidos o patrocínio e a infra-estrutura de melhoria dos processos; (ii) diagnóstico, na qual são realizadas avaliações e recomendações para a melhoria de processos, (iii) estabelecimento, onde é construída a infra-estrutura de melhoria dos processos, (iv) ação, quando as melhorias planejadas são implementadas; e (v) lições, na qual são analisadas as lições aprendidas do esforço de melhoria do processo de software, resultando em atualizações dos processos de software.

Neste trabalho utilizou-se a fase de Diagnóstico que é composta das seguintes etapas:

- Caracterizar o estado corrente e desejado - na qual é utilizado um modelo de referência para, a partir da identificação do estado atual, definir o estado desejado. É como identificar o início e o fim da jornada. 


\section{Simpósio Brasileiro de Qualidade de Software}

- Desenvolver recomendações - na qual desenvolvem-se recomendações, sugerindo meios de como proceder nas atividades subseqüentes. As atividades desta fase são desempenhadas por pessoas mais experientes ou por especialistas relevantes na organização. Suas recomendações são baseadas nas decisões realizadas pelos gerentes chefes e patrocinadores.

\section{Modelo de Referência - CMMI}

O CMMI constitui em um framework de melhoria de processo que integra várias disciplinas sendo formado por uma combinação de modelos.

O CMMI apresenta representações distintas: estágio e contínua. A representação estágio é basicamente a mesma apresentada em outros modelos do SEI, onde cada Área de Processo se associa a um dos 5 (cinco) níveis de maturidade.

Já na representação contínua, os níveis de maturidade não existem, passando a possuir 6 (seis) níveis de capacidade. Esta representação também possui uma divisão em categorias, sendo elas: Gerenciamento de Projeto, Gerenciamento de Processo, Engenharia e Suporte. As duas áreas de processo abordadas neste artigo fazem parte da categoria de Engenharia, a saber: Desenvolvimento de Requisitos e Gerência de Requisitos.

A área de processo de Desenvolvimento de Requisitos define um conjunto completo de requisitos do cliente a ser usado no desenvolvimento dos requisitos do produto, os requisitos de produto e componentes de produto para utilização no design dos produtos e componentes. Além disso, realiza uma análise para definir, derivar e entender os requisitos.

A área de processo de Gerenciamento de Requisitos descreve as atividades para obter e controlar as mudanças de requisitos e assegurar a atualização dos planos do projeto e dados relevantes. Fornece a rastreabilidade dos requisitos do cliente para o produto e para o componente do produto. Assim, gerenciar requisitos é realizar uma sequiência de eventos dinâmicos e freqüentemente recursivos, sendo os mesmos fundamentais para um processo de engenharia controlado e disciplinado.

\section{Método de Avaliação SCAMPI}

O método de avaliação $S \boldsymbol{C A M P \boldsymbol { I } ^ { S M }}$ é utilizado para identificar pontos fortes, fracos e classificar a realização das práticas específicas e genéricas referentes ao $\boldsymbol{C M M I}$ [SCAMPI ${ }^{S M}$, 2001]. Para tanto, são verificados os Indicadores de Implementação Prática (PII - Practice Implementation Indicators), sendo: (a) artefatos diretos, os quais são a finalidade da execução prática - sem a produção desses a prática não é "conforme"; (b) artefatos indiretos, auxiliam a execução prática, porém não são a finalidade da mesma - especialmente úteis quando há alguma dúvida sobre a realização da prática; (c) afirmações, declarações orais ou escritas que confirmam ou que suportam a execução de uma prática específica ou genérica.

Baseado nos dados dos PII é realizada a caracterização da implementação de cada prática do modelo $\boldsymbol{C M M I}$. A Tabela 1 apresenta os tipos de caracterização da implementação prática. 
Tabela 1. Caracterização da Implementação Prática [SCAMPI ${ }^{S M}$, 2001]

\begin{tabular}{|l|l|}
\hline \multicolumn{2}{|c|}{ Caracterização da Implementação Prática } \\
\hline $\begin{array}{l}\text { Completamente Implementada (FI - } \\
\text { Fully Implemented })\end{array}$ & $\begin{array}{l}\text { Apresenta Artefato(s) Direto(s) adequado(s); } \\
\text { É suportado por Artefato Indireto e/ou Afirmação; } \\
\text { Não é notada nenhuma fraqueza. }\end{array}$ \\
\hline $\begin{array}{l}\text { Implementada em Grande Parte (LI - } \\
\text { Largely Implemented })\end{array}$ & $\begin{array}{l}\text { Apresenta Artefato(s) Direto(s) adequado(s); } \\
\text { É suportado por Artefato Indireto e/ou Afirmação; } \\
\text { Uma ou mais fraquezas são notadas. }\end{array}$ \\
\hline $\begin{array}{l}\text { Parcialmente Implementada } \\
(\boldsymbol{P I}-\boldsymbol{P a r t i a l l y} \text { Implemented })\end{array}$ & $\begin{array}{l}\text { Falta de Artefato(s) Direto(s) ou julgado(s) inadequado(s); } \\
\text { Artefato(s) Indireto(s) e/ou Afirmação(ões) indicam que } \\
\text { alguns aspectos da prática são implementados; }\end{array}$ \\
\hline $\begin{array}{l}\text { Não Implementada } \\
(\text { NI }- \text { Not Implemented })\end{array}$ & $\begin{array}{l}\text { Qualquer situação não coberta pelo mencionado } \\
\text { anteriormente. }\end{array}$ \\
\hline
\end{tabular}

\section{Diagnóstico da Área de Requisitos}

A Empresa Brasília está no mercado de desenvolvimento de software a aproximadamente 5 (cinco) anos. A empresa, de médio porte, possui atualmente a certificação ISO 9001 e conta com um processo iterativo, concebido a partir das Áreas Chaves de Processo do nível 2, preconizadas pelo modelo de maturidade $S W$ - $C M M$, do Instituto de Engenharia de Software, e a partir das disciplinas e atividades do RUP Rational Unified Process.

Em conformidade com os objetivos estabelecidos pela Empresa Brasília, o cenário alvo desta pesquisa é a área de requisitos da fábrica de software composta por 10 (dez) analistas de requisitos, distribuídos em diferentes projetos. Desses profissionais, alvos da pesquisa, 6 (seis) participaram representando a avaliação de 3 (três) projetos distintos, os quais são denominados neste artigo de Projeto "A", Projeto "B" e Projeto "C". Definidos os métodos utilizados e o alvo da pesquisa, foram planejadas as atividades a serem executas de acordo com o ciclo de melhoria definido pela Empresa Brasília. A seguir são apresentadas as duas etapas realizadas na fase de Diagnóstico.

\subsection{Caracterização do Estado Atual da Empresa - Etapa I da Fase de Diagnóstico}

Dando início a primeira etapa da fase de Diagnóstico, foi realizada a coleta de dados a partir das seguintes atividades:

- Observação participativa: onde o pesquisador esteve in loco presenciando e participando dos fatos e analisando documentos.

- Elaboração do Questionário: desenvolvido com base no questionário de maturidade [ZUBROW, 1994], possui o intuito de auxiliar as entrevistas. Dividido por Área de Processo - Desenvolvimento de Requisitos e Gerenciamento de Requisitos - para a montagem e estruturação do questionário foram analisadas as práticas das metas específicas, do modelo $C M M I$, e elaboradas de 2 a 4 questões, para cada. As duas seções citadas anteriormente possuem em sua introdução, um texto explicativo referente aos objetivos da Área de Processo em questão. A Figura 1 apresenta parte do questionário desenvolvido. 
- Realização de entrevistas semi-estruturadas: estruturadas de forma a obter informações sobre a implementação das práticas propostas pelo modelo de referência e com o apoio do questionário, as entrevistas foram realizadas individualmente e tiveram um tempo médio de duração de 60 minutos.

- Elaboração da Planilha Individual de Avaliação de Projeto (PIAP): construção de uma planilha com o intuito transcrever e consolidar os dados obtidos durante as entrevistas. Essa planilha foi aplicada a cada um dos projetos avaliados.

\section{GERENCIAMENTO DE REQUISITOS}

\begin{tabular}{l}
\hline \hline A Área de Processo Gerenciamento de Requisitos do CMMI descreve as atividades para obter e controlar as \\
mudanças de requisitos e assegurar que planos do projeto e dados relevantes estejam mantidos atuais. Fornece a \\
rastreabilidade dos requisitos do cliente para o produto e para o componente do produto. O Gerenciamento dos \\
Requisitos assegura-se de que as mudanças nos requisitos estejam refletidas em planos do projeto, em atividades, e \\
em produtos do trabalho. Este ciclo de mudanças pode afetar todas as áreas restantes do processo de engenharia; \\
assim o Gerenciamento de Requisitos é uma sequiência de eventos dinâmicos e freqüentemente recursivos, sendo o \\
mesmo fundamental para um processo de projeto de engenharia controlado e disciplinado.
\end{tabular}

1. GERÊNCIA DE REQUISITOS

1.1. Obter o entendimento dos requisitos

\begin{tabular}{|c|c|c|c|c|c|c|}
\hline & & Sim & Não & $\begin{array}{l}\text { Não se } \\
\text { aplica }\end{array}$ & $\begin{array}{l}\text { Não } \\
\text { sei }\end{array}$ & $\begin{array}{l}\text { Índice } \\
\text { realizado } \\
{[1 \text { a } 9]}\end{array}$ \\
\hline A. & $\begin{array}{l}\text { Critérios que identificam os canais e fontes oficiais para a obtenção } \\
\text { dos requisitos são estabelecidos e documentados? }\end{array}$ & () & () & () & () & () \\
\hline B. & $\begin{array}{l}\text { Critérios de aceitação dos requisitos são estabelecidos e } \\
\text { documentados? }\end{array}$ & () & ( ) & () & () & ( ) \\
\hline C. & $\begin{array}{l}\text { Atividades para a análise dos requisitos obtidos são desenvolvidas } \\
\text { antes de realizado o acordo acerca do conjunto de requisitos a serem } \\
\text { cumpridos? }\end{array}$ & () & ( ) & ( ) & () & ( ) \\
\hline
\end{tabular}

Figura 1. Parte do questionário aplicado referente à PA Gerenciamento de Requisitos

Após a coleta de dados, deu-se a caracterização da implementação das práticas, a partir da análise dos dados obtidos (PII), sendo os mesmos documentados na Planilha de Avaliação Consolidada da Área de Requisitos (PLACAR). A Figura 2 apresenta uma parte da PLACAR, referente ao Gerenciamento de Requisitos. Neste exemplo, é demonstrada a caracterização da implementação prática de cada projeto em relação a cada prática e meta específica. Na última coluna, são exibidos os dados que caracterizam o Gerenciamento de Requisitos, da Empresa Brasília.

\begin{tabular}{|c|c|c|c|c|c|}
\hline & & $\begin{array}{c}\text { Projeto } \\
\text { A }\end{array}$ & $\begin{array}{c}\text { Projeto } \\
\text { B } \\
\end{array}$ & $\begin{array}{c}\text { Projeto } \\
\text { C }\end{array}$ & Resultado \\
\hline SG 1 & GERENCIAR REQUISITOS & & & & \\
\hline SP 1.1-1 & Obter o entendimento dos requisitos & NI & NI & NI & NI \\
\hline SP 1.2-2 & Obter o comprometimento dos participantes & PI & PI & PI & PI \\
\hline SP 1.3-1 & Gerenciar mudanças nos requisitos & PI & PI & PI & PI \\
\hline SP 1.4-2 & Manter a rastreabilidade bi-direcional dos requisitos & PI & PI & PI & PI \\
\hline SP 1.5-1 & Identificar inconsistências entre trabalho do projeto e os requisitos & NI & NI & NI & NI \\
\hline & & PI & PI & PI & PI \\
\hline
\end{tabular}

Figura 2. Parte da PLACAR referente ao Gerenciamento de Requisitos 


\section{Simpósio Brasileiro de Qualidade de Software}

Finalizada a coleta de dados e a caracterização da implementação das práticas, constatou-se que, das 17 (dezessete) práticas específicas avaliadas nenhuma estava completamente implementada, apenas 1 (uma) estava largamente implementada, 14 (quatorze) estavam parcialmente implementadas e 2 (duas) não estavam implementadas.

A partir destas constatações foi realizada uma atividade para a identificação de pontos fracos, suas causas e impactos no processo de desenvolvimento de software da Empresa Brasília. As Tabelas 2 e 3 apresentam alguns pontos identificados.

Tabela 2. Pontos Fracos, Causas e Impactos no Gerenciamento de Requisitos

\begin{tabular}{|c|c|c|c|}
\hline ID & Ponto Fraco & Causa & Impacto \\
\hline \multicolumn{4}{|c|}{ SG 1/ SP 1.1-1 Obter o entendimento dos requisitos } \\
\hline 1 & $\begin{array}{l}\text { Falta de critérios a } \\
\text { serem utilizados na } \\
\text { identificação de } \\
\text { stakeholders } \\
\text { relevantes. }\end{array}$ & $\begin{array}{l}\text { Não foram identificados no processo da } \\
\text { empresa atividades, procedimentos, ou } \\
\text { diretrizes que definissem e documentassem } \\
\text { critérios para a identificação de } \\
\text { stakeholders relevantes. }\end{array}$ & $\begin{array}{l}\text { Realização do trabalho com } \\
\text { stakholders inadequados. }\end{array}$ \\
\hline 2 & $\begin{array}{l}\text { Falta de critérios a } \\
\text { serem utilizados na } \\
\text { aceitação dos } \\
\text { requisitos. }\end{array}$ & $\begin{array}{l}\text { Não foram identificados no processo da } \\
\text { empresa atividades, procedimentos, ou } \\
\text { diretrizes que definissem e documentassem } \\
\text { os critérios para a aceitação de requisitos. }\end{array}$ & $\begin{array}{l}\text { Possibilidade de aceite de } \\
\text { requisitos que não são } \\
\text { requisitos e de requisitos } \\
\text { que podem não ser } \\
\text { verificados, por exemplo. }\end{array}$ \\
\hline 3 & $\begin{array}{l}\text { Falta de documentação } \\
\text { da atividade de pré- } \\
\text { análise de requisitos } \\
\text { antes dos mesmos } \\
\text { serem aceitos. }\end{array}$ & $\begin{array}{l}\text { Não foram identificados no processo da } \\
\text { empresa atividades, procedimentos, ou } \\
\text { diretrizes que definissem e documentassem } \\
\text { uma atividade de pré-análise dos } \\
\text { requisitos, antes do seu aceite. }\end{array}$ & $\begin{array}{l}\text { Possibilidade de aceite de } \\
\text { requisitos que não podem } \\
\text { ser realizáveis no prazo, } \\
\text { custo e esforço estimados. }\end{array}$ \\
\hline
\end{tabular}

Tabela 3. Pontos Fracos, Causas e Impactos no Desenvolvimento de Requisitos

\begin{tabular}{|c|l|l|l|}
\hline ID & \multicolumn{1}{|c|}{ Ponto Fraco } & \multicolumn{1}{c|}{ Causa } & \multicolumn{1}{c|}{ Impacto } \\
\hline \multicolumn{2}{|c|}{ SG / SP 1.1-1 Coletar necessidades dos stakeholders } \\
\hline $\mathbf{1}$ & $\begin{array}{l}\text { Falha na comunicação } \\
\text { do planejamento das } \\
\text { atividades de projeto } \\
\text { aos integrantes da } \\
\text { equipe. }\end{array}$ & $\begin{array}{l}\text { Não foram identificados no processo da } \\
\text { empresa atividades, procedimentos, ou } \\
\text { diretrizes que definissem e documentassem } \\
\text { a comunicação do planejamento das } \\
\text { atividades de projeto aos integrantes da } \\
\text { equipe. }\end{array}$ & $\begin{array}{l}\text { Possibilidade de } \\
\text { componentes da equipe } \\
\text { ficarem sem atividade ou } \\
\text { realizarem atividades que } \\
\text { não são relevantes. }\end{array}$ \\
\hline \multirow{2}{*}{ SG 1 / SP 1.1-2 Elicitar necessidades dos stakeholders } \\
\hline $\begin{array}{l}\text { Falta de elicitação das } \\
\text { necessidades, } \\
\text { expectativas e } \\
\text { restrições dos } \\
\text { stakeholders (equipe } \\
\text { interna). }\end{array}$ & $\begin{array}{l}\text { Não foram identificados no processo da } \\
\text { empresa atividades, procedimentos, ou } \\
\text { diretrizes que definissem e documentassem } \\
\text { a elicitação das necessidades, expectativas } \\
\text { e restrições dos stakeholders (equipe } \\
\text { interna). }\end{array}$ & $\begin{array}{l}\text { Possibilidade de problemas } \\
\text { durante o projeto devido à } \\
\text { falta de conhecimento das } \\
\text { necessidades, expectativas e } \\
\text { restrições dos stakeholders } \\
\text { (equipe interna). }\end{array}$ \\
\hline
\end{tabular}




\section{Simpósio Brasileiro de Qualidade de Software}

\subsection{Definição das Recomendações - Etapa II da Fase de Diagnóstico}

A partir dos pontos fracos, causas e impactos identificados foram estabelecidas recomendações necessárias para a melhoria do processo de desenvolvimento de software da Empresa Brasília.

A seguir são apresentadas algumas destas recomendações para a melhoria do processo de Gerenciamento e Desenvolvimento de Requisitos:

I. Definir e documentar critérios para a identificação dos stakeholders relevantes ao projeto. Estes critérios devem auxiliar a execução da atividade de identificação dos stakeholders.

II. Definir e documentar critérios de aceitação dos requisitos.

III. Inserir no processo uma atividade para identificar e registrar a volatilidade dos requisitos a partir do seu histórico de alterações.

IV. Inserir no processo uma atividade de manutenção de matrizes de rastreabilidade.

V. Estabelecer uma política de comunicação com o intuito de minimizar falhas de conversação entre equipes e integrantes.

VI. Criar um novo documento ou uma seção dentro de um artefato já existente, por exemplo, Documento de Visão ou Plano de Trabalho, para registrar a elicitação das necessidades, expectativas e restrições dos stakeholders (equipe interna).

VII. Disseminar a utilização do artefato "Lista de Problemas", definido pelo processo de desenvolvimento da Empresa Brasília, entre os envolvidos e apresentar sua importância.

VIII. Inserir no processo uma atividade de manutenção do relacionamento e dependência entre os requisitos.

\section{Conclusão}

A realização da fase de Diagnóstico produziu como principal benefício, para a empresa Brasília, a identificação dos pontos fracos que inviabilizavam a prática bem estabelecida da Gerência e Desenvolvimento de Requisitos. Nessa fase, o processo da empresa foi caracterizado como sendo não executado ou executado parcialmente, devido a uma ou mais metas específicas propostas pelo modelo $\boldsymbol{C M M I}$ não terem sido satisfeitas.

A utilização da abordagem IDEAL, do modelo de referência $\boldsymbol{C M M I}$, da metodologia de avaliação $\boldsymbol{S C A M P I}{ }^{S \boldsymbol{M}}$, e dos artefatos auxiliares - questionário, PIAP e PLACAR - da forma que foi apresentada neste artigo, não se limita á empresa Brasília, podendo ser aplicado a qualquer outra organização de desenvolvimento de software, mesmo que esta escolha outras áreas de processo para iniciar seu programa de melhoria de processos.

Dando continuidade a este trabalho, implantou-se a fase de Elaboração da IDEAL [CORRÊA et al, 2004]. Nessa fase foi definido um conjunto de instruções que norteia a empresa em direção à melhoria dos processos de gerenciamento e desenvolvimento de requisitos, no que tange a execução completa das práticas específicas propostas pelo modelo $\boldsymbol{C M M I}$. 


\section{Simpósio Brasileiro de Qualidade de Software}

A partir da implantação das diretrizes propostas, a Empresa Brasília almeja melhorar significativamente o processo realizado atualmente pela área de requisitos e dar prosseguimento com a execução das próximas fases da abordagem IDEAL: elaborar e implementar o plano de ações; revisar e analisar as lições aprendidas e incorporá-las ao processo, e assim, iniciar um novo ciclo de melhorias para as demais práticas do CMMI.

\section{Referências}

Carvalho, A. E. S., Tavares, H. C., Castro, J. B., (2001) "Uma Estratégia para a Implantação de uma Gerência de Requisitos Visando a Melhoria dos Processos de Software", IV Workshop em Requisitos, Buenos Aires, Argentina.

Capability Maturity Model ${ }^{\circledR}$ Integration $\left(\mathrm{CMMI}^{\mathrm{SM}}\right)$ (2001), Version 1.1 “CMMI ${ }^{\mathrm{SM}}$ for Systems Engineering, Software Engineering, Integrated, Product and Process Development, and Supplier Sourcing (CMMI-SE/SW/IPPD/SS, V1.1)”.

Corrêa, G. M., Figueiredo, R. M. C., Oliveira, K. M., Araújo, J. M. P., (2004) "Diretrizes para a melhoria da Gerência e Desenvolvimento de Requisitos em uma Empresa de Software", VI Simpósio Internacional de Melhoria de Processo de Software, São Paulo, Brasil.

Deming, W. E. (1990) "Qualidade - A Revolução da Administração", Rio de Janeiro, Saraiva.

Mcfeeley, B. (1996) "IDEAL ${ }^{\text {SM }}$ : A User's Guide for Software Process Improvement" Handbook CMU/SEI-96-HB-001, Software Engineering Institute, Carnegie Mellon University, Pittsburgh, Pennsylvania 15213

Members Of The Assessment Method Integrated Team Standard CMMI ${ }^{\mathrm{SM}}$ Appraisal Method for Process Improvement (SCAMPI $^{\mathrm{SM}}$ ) (2001), Version 1.1: Method Definition Document.

Pires, C. G., Marinho, F., Telles, G. Belchior, A., (2004) "A Experiência de Melhoria do Processo do Instituto Atlântico Baseado no SW-CMM nível 2”, III Simpósio Brasileiro de Qualidade de Software, Brasília, Brasil.

Santos, D. A. S., Pimentel, B. S., Machado F. T., Cardoso, G. S., Filho, W. P. P., (2004) "Personalização e Implantação de Procedimentos de Gestão da Qualidade dentro de um Programa de Melhoria", III Simpósio Brasileiro de Qualidade de Software, Brasília, Brasil.

Sommervile, I., Ransom, J., (2005) “An Empirical Study of Industrial Requirements Engineering Process Assessment and Improvement", ACM Transactions on Software Engineering and Methodology, Vol. 14, No. 1, January 2005, Pages 85-117.

Zubrow, D., Hayes, W., Siegel, J., Goldenson, D., (1994) - Maturity Questionnaire Special Report, CMU/SEI-94-SR-7 - Empirical Methods, ON-LINE. Disponível em: http://www.sei.cmu.edu/pub/documents/94.reports/pdf/sr07.94.pdf 